University of Wollongong

Research Online

Faculty of Engineering - Papers (Archive)

Faculty of Engineering and Information

Sciences

$1-1-2010$

Gapless insulator and a band gap scaling law in semihydrogenated graphene

Anthony Wright

arw75@uow.edu.au

TE. O'Brien

School of Engineering Physics Univ of Wollongong

Chao Zhang

University of Wollongong, czhang@uow.edu.au

Dieter Beaven

djdb684@uow.edu.au

Follow this and additional works at: https://ro.uow.edu.au/engpapers

Digitart of the Engineering Commons

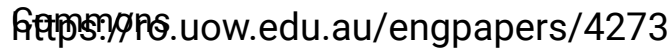

Network

Logo

Recommended Citation

Wright, Anthony; O'Brien, T E.; Zhang, Chao; and Beaven, Dieter: Gapless insulator and a band gap scaling law in semihydrogenated graphene 2010, 043104-1-043104-3.

https://ro.uow.edu.au/engpapers/4273

Research Online is the open access institutional repository for the University of Wollongong. For further information contact the UOW Library: research-pubs@uow.edu.au 


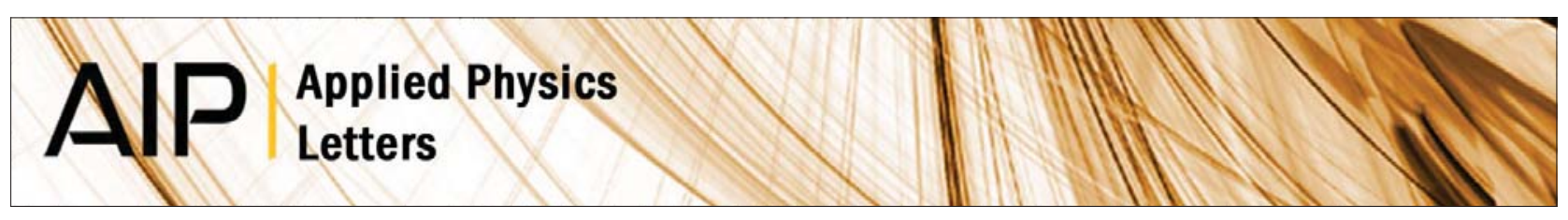

\section{Gapless insulator and a band gap scaling law in semihydrogenated graphene}

A. R. Wright, T. E. O'Brien, D. Beaven, and C. Zhang

Citation: Appl. Phys. Lett. 97, 043104 (2010); doi: 10.1063/1.3469941

View online: http://dx.doi.org/10.1063/1.3469941

View Table of Contents: http://apl.aip.org/resource/1/APPLAB/v97/i4

Published by the American Institute of Physics.

\section{Related Articles}

A density functional theory study of epitaxial graphene on the (3×3)-reconstructed C-face of SiC Appl. Phys. Lett. 102, 093101 (2013)

Magnetic and electronic properties of Fe3O4/graphene heterostructures: First principles perspective J. Appl. Phys. 113, 083711 (2013)

Inverse relationship between carrier mobility and bandgap in graphene

J. Chem. Phys. 138, 084701 (2013)

Directional quantum transport in graphyne $\mathrm{p}-\mathrm{n}$ junction J. Appl. Phys. 113, 073710 (2013)

Electronic screening in stacked graphene flakes revealed by scanning tunneling microscopy Appl. Phys. Lett. 102, 053116 (2013)

\section{Additional information on Appl. Phys. Lett.}

Journal Homepage: http://apl.aip.org/

Journal Information: http://apl.aip.org/about/about_the_journal

Top downloads: http://apl.aip.org/features/most_downloaded

Information for Authors: http://apl.aip.org/authors

\section{ADVERTISEMENT}

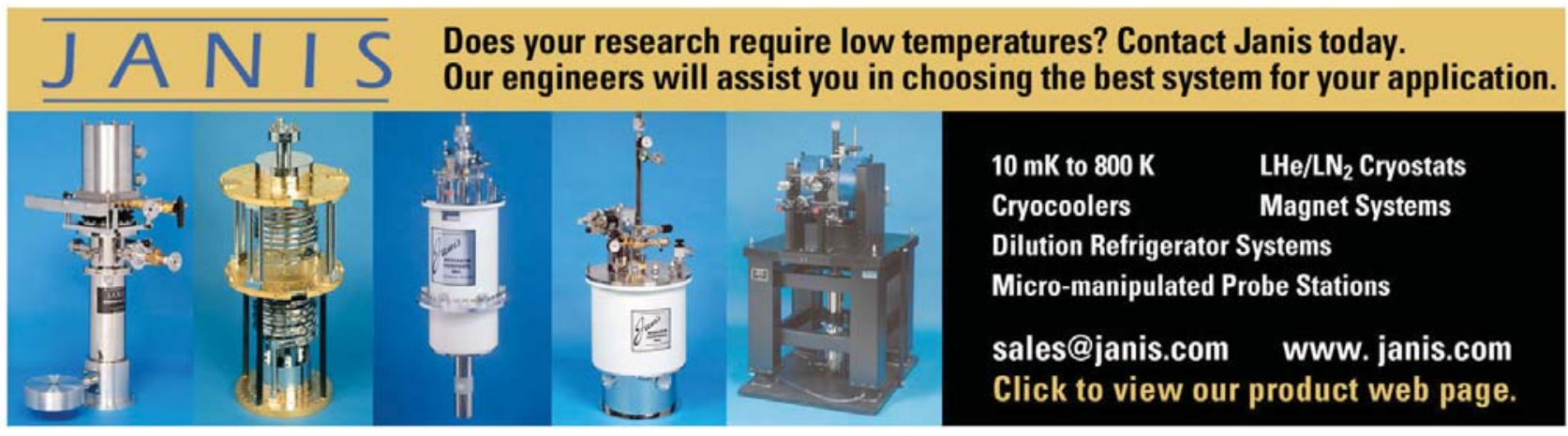




\title{
Gapless insulator and a band gap scaling law in semihydrogenated graphene
}

\author{
A. R. Wright, ${ }^{1}$ T. E. O'Brien, ${ }^{1}$ D. Beaven, ${ }^{1,2}$ and C. Zhang ${ }^{1, a)}$ \\ ${ }^{1}$ School of Engineering Physics, University of Wollongong, New South Wales 2552, Australia \\ ${ }^{2}$ School of Computer Science and Software Engineering, University of Wollongong, \\ New South Wales 2552, Australia
}

(Received 18 June 2010; accepted 7 July 2010; published online 26 July 2010)

\begin{abstract}
We demonstrate two unusual electronic properties of semihydrogenated graphene with variable sized A- or B-hydrogenated domains within the tight-binding formalism as follows: (i) a universal band gap scaling law which states that the band gap depends linearly upon the ratio of the number of A- to B-hydrogenated atoms, $N_{A} / N_{B}$, reaching zero gap at $N_{A}=N_{B}$, but independent of the domain size, and (ii) an insulating state with zero band gap at $N_{A}=N_{B}$, a rare phenomenon in nature. We confirm this gapless insulator state by the zero optical conductance at low frequencies. (C) 2010 American Institute of Physics. [doi:10.1063/1.3469941]
\end{abstract}

Graphene is a zero-band gap semiconductor whose $E$ $-k$ relation is linear near the corners of the two-dimensional (2D) hexagonal Brillouin zone, leading to vanishing effective mass for electrons and holes. ${ }^{1}$ The observation of the quantum Hall effect in graphene provided direct evidence of the theoretically predicted $\pi$ Berry's phase of massless Dirac fermions. ${ }^{2}$ The massless neutrino-like energy spectrum of electrons and holes in graphene can lead to specific features of the transport properties such as the minimum dc conductivity of $\sigma_{\min }=4 e^{2} / h^{3}$

More recently, attention has been paid to a graphene sheet which was hydrogenated to form graphane, which has semiconducting properties. The band gap calculated via local-density approximation is $3.5 \mathrm{eV}$ (Ref. 4) while the GW calculation gives a value of $5.4 \mathrm{eV}{ }^{5}$ Furthermore, it has been hypothesized that a semihydrogenated version of graphene could be created via the blocking of a substrate, or the application of an external electric field, removing the hydrogen atoms from one side. ${ }^{6}$ In this form, both ferromagnetic order ${ }^{7}$ and the opening of a band gap ${ }^{6}$ have been predicted due to the breaking of symmetry between hydrogen-bonded and nonbonded carbon atoms. A detection scheme for valley polarization in graphene systems with broken symmetry has been demonstrated, ${ }^{8}$ and it has been further shown that due to this inequivalence, the universal conductance breaks down at low frequencies.

The characterization of graphene-based materials via the optical conductivity has great potential due to the various "breakdowns" and resonances observed for energies up to the optical regime. Furthermore, the optical properties of graphene within the terahertz to far-infrared regime-a technologically important region of the electromagnetic spectrum-has become quite a hot topic. It has been shown that the universal conductivity of single layer graphene ${ }^{10}$ is robust until well over $1 \mathrm{eV},{ }^{11,12}$ however this is no longer the case for graphene bilayers. ${ }^{13-15}$ For single layer graphene the optical conductivity can be altered by creating antidots, ${ }^{15}$ stretching, ${ }^{16,17}$ and high intensity fields. ${ }^{18}$ When restricting the geometry to that of a pseudo-one-dimensional ribbon, the universal conductance is destroyed completely, being re-

${ }^{a)}$ Electronic mail: czhang@uow.edu.au. placed by resonant peaks corresponding to van-Hove singularities in the density of states. ${ }^{19,20}$ This trend of exhibiting vastly different optical properties depending on geometry and field strength makes graphene-based materials a prime candidate for photonic applications, and makes characterization of different structures via such a non-destructive method as optical transmittance feasible.

In this paper, we shall reveal two unusual and remarkable properties of semihydrogenated graphene as follows: a band gap scaling law and a gapless insulating state. We find that by creating domains of different hydrogenation configurations, the band gap variation is governed by a scaling law in which the domain size is irrelevant and only the ratio of A-B pairs in different hydrogenation configurations matters. We calculate the optical conductance of different configurations, and show a relationship between the band gap size and the initial peak magnitude. The single domain semihydrogenated single layer graphene has the largest band gap which is equal to the onsite energy of the hydrogenated atom. When the A-domain size is equal to the B-domain size, i.e., $N_{A}$ $=N_{B}$, the system is a zero gap material. However, the electronic transition from the lower band to the upper band is forbidden due to the presence of hydrogen atoms. Therefore it is an insulator with zero band gap. This property is confirmed by the vanishing optical conductance at low energy. Therefore by alternatively hydrogenating A and B sublattices, graphene changes from a gapless semiconductor to a gapless insulator.

Our model system is depicted in Fig. 1. We assume that graphene on a substrate or exposed to an electric field becomes semihydrogenated such that only A (or B) atoms accept a hydrogen atom. However we also assume that on initial exposure to hydrogen, different regions of the sample will become hydrogenated on different lattice sites, thus forming domains of both $\mathrm{A}$ and $\mathrm{B}$ hydrogenation. Between these domains we expect that there will be an A-B pair where either both or neither are hydrogenated. In our model we have assumed the former, however this detail is irrelevant to our results. We further assume that the most energetically favorable configuration for these domains is that they will be hexagonal. Therefore we construct our unit cell as shown in Fig. 1, where there are two characteristic lengths as follows: 


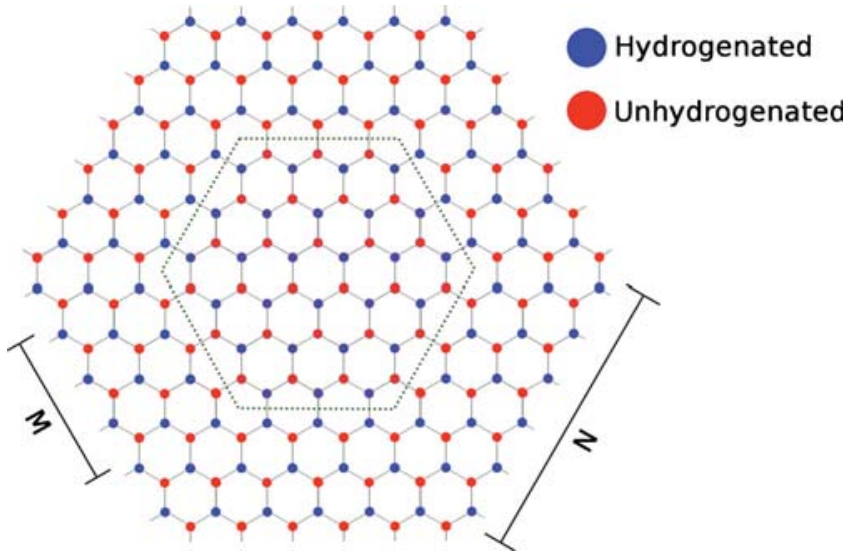

FIG. 1. (Color online) A schematic illustration of the predicted domain geometry for semihydrogenated graphene. The inner hexagon has been A-hydrogenated, and the outer region has been B-hydrogenated. This means that there is a ring between the two domains where nearest neighbors are both A- or both B-hydrogenated.

the side length $\mathrm{S}$ and an inner length $\mathrm{L}$. The number of atoms in the unit cell is given by $N=6 N_{L} S^{2}$, where $N_{L}$ is the number of layers. The number of A-B pairs in the inner A-hydrogenated domain is then $6 L^{2}$, and the number of A-B pairs in the outer B-hydrogenated domain is $6\left(S^{2}-L^{2}\right)$.

We calculate the electronic properties via the tightbinding approximation. The Hamiltonian is given by

$$
H=\sum_{i} \Delta_{i} c_{i}^{\dagger} c_{i}+\sum_{i, j} t_{i, j} c_{i}^{\dagger} c_{j},
$$

where $\Delta_{i}$ is the onsite energy (at site $i$ ) caused by the hydrogen atom, and $t_{i j}=t=3 \mathrm{eV}$ if $i$ and $j$ are first nearest neighbors, and is zero otherwise. If there is a hydrogen atom present, we choose $\Delta_{i}=\Delta_{H}=0.1 t$, and if not, $\Delta_{i}=0$.

The band structures for the single layer case are shown in Fig. 2 for $S=4$ and $L=[0,3] . L=S$ is equivalent to $L=0$. The main differences between the band structures occur at small energies. Some degeneracy is lifted near the $K$ points, and when moving along directions of low symmetry (e.g., $\left.K^{\prime} \rightarrow K\right)$. The most interesting feature however, is the variation in the band gap as a function of inner length $L$. It can be seen that the band gap for $L=3$ is almost zero. As we will see below, this is because $L^{2} / S^{2}=3^{2} / 4^{2} \approx 0.5$.

In Fig. 3 we plot the band gaps $(\Delta)$ for all possible $L$ with $S=[2,10]$, as a function of $L^{2} / S^{2}$. It can be seen that the relationships are quite predictable

$$
\frac{\Delta}{\Delta_{H}}=\left|1-2 \frac{S^{2}}{L^{2}}\right| \text {. }
$$

It should be noted that this is a function of the regular geometry which we have assumed. For randomly allocated hydrogen, the band gaps become randomly spaced between $\Delta=0$ and $\Delta=\Delta_{H}$. In this case the low energy band structures also become less smooth than those of Fig. 2, and more degeneracies are lifted at the high symmetry points.

It is statistically reasonable to assume that for infinite semihydrogenated graphene, half the carbon atoms will be part of an A-hydrogenated domain, and half will be B-hydrogenated. For this reason, we would expect infinite semihydrogenated single layer graphene to be a zero-gap material just like ordinary graphene. If this is the case, how would one detect whether the graphene has become hydro-

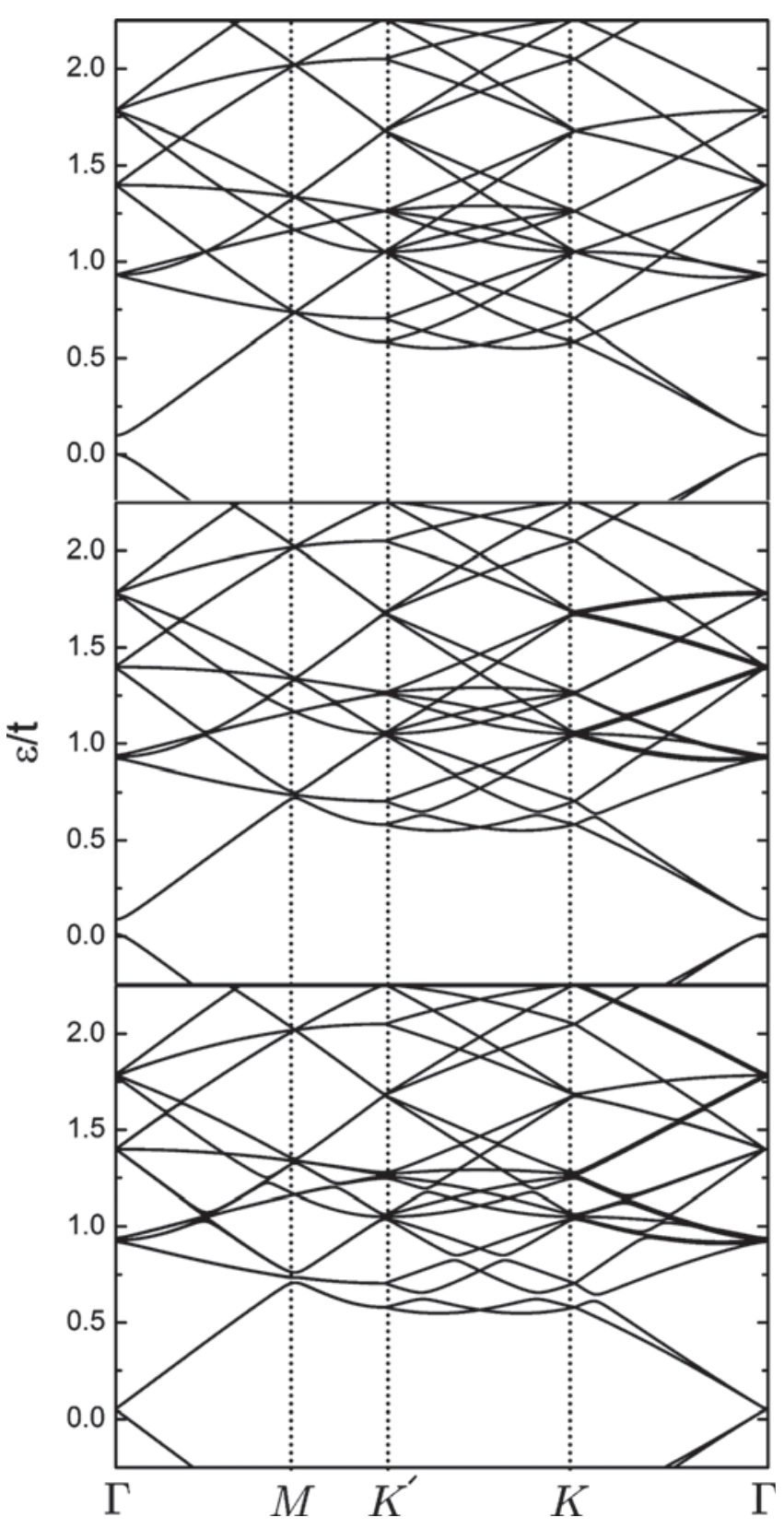

FIG. 2. The band structure of the $S=4$ semihydrogenated graphene structures, with $L=0,1,2$ presented down the page, respectively. The most noticeable differences between different inner lengths can be seen at low energies with the vanishing of the finite gap at the $\Gamma$ point, and the lifting of some degeneracies around the $K$ points. Note that the Dirac point corresponds to the $\Gamma$ point and not the $K$ points as in usual graphene. This is an artifact of the construction of our unit cell.

genated or not? With this question in mind we proceed to calculate the optical conductivity of our model semihydrogenated graphene. The Kubo formula for optical conductivity is given as $^{21}$

$$
\sigma_{\mu, \nu}(\omega)=\frac{1}{\omega} \int_{0}^{\infty} \mathrm{d} t e^{i \omega t}\left\langle\left[J_{\mu}(t), J_{\nu}(0)\right]\right\rangle .
$$

Here the components of the current operator can be calculated from $J_{\nu, \mu}(t)=e^{i H t} J_{\nu, \mu}(0) e^{-i H t}$, where $J_{\nu, \mu}(0)$ $=\Psi^{\dagger}(\mathbf{r}) \hat{v}_{\nu, \mu} \Psi\left(\mathbf{r}^{\prime}\right)$, in which $\hat{v}_{\nu, \mu}=\partial H / \partial k_{\nu, \mu}$, and $\nu, \mu=x, y$.

In Fig. 4 we present the results for $S=4$ semihydrogenated graphene, with $L=[0,3]$. The case $L=0$ corresponds to regular semihydrogenated graphene, and has been reported 


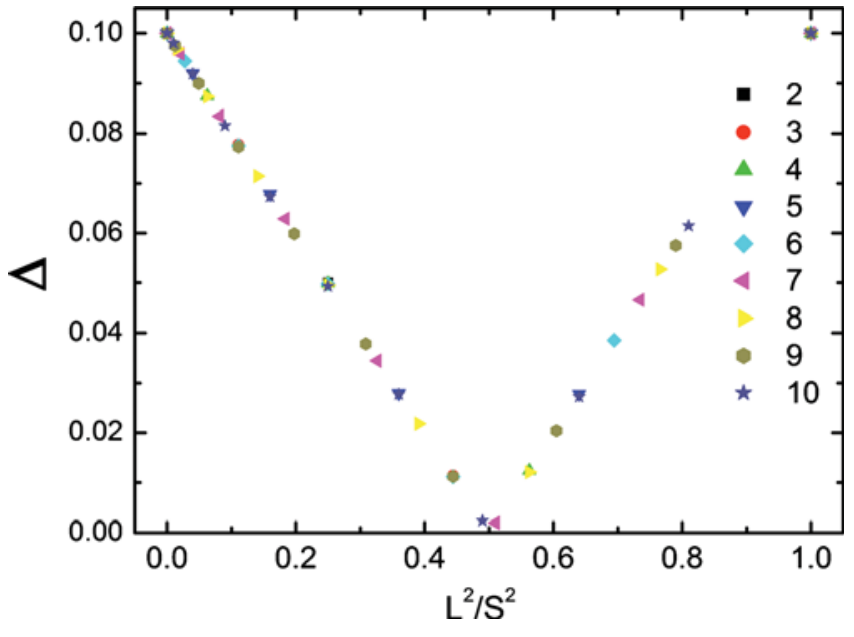

FIG. 3. (Color online) The variation in the band gap for single layer semihydrogenated graphene as a function of $L^{2} / S^{2}$, which is proportional to the fraction of A-hydrogenated atoms. A zero gap occurs when $N_{A}=N_{B}$.

previously. ${ }^{9}$ The $L=0$ result is characterized by the peculiar universal property that $\sigma_{\Delta}=2 \sigma_{0}$, that is, when $\hbar \omega / t=\Omega=\Delta$, the optical conductivity is precisely twice the universal conductivity of graphene. However, we find that as the band gap goes to zero, this phenomenal result disappears, and the onset conductivity approaches $\sigma_{0}$.

These results are particularly curious however, as we see that even though semihydrogenated graphene with $(S, L)$ $=(4,3)$ is an almost zero-gap material, interband optical transitions are forbidden, and the optical conductance is zero for $\hbar \omega / t<0.9 \Delta$. It is known that the immediate effect of semihydrogenation is the broken inversion symmetry of the $2 \mathrm{D}$ honeycomb lattice. ${ }^{6,7}$ This broken inversion symmetry in the crystal structure leads to a change in the wave function symmetry required for the interband optical transition. As a result, the optical conductance vanishes even though the band gap has been diminished, and we have a potential zero-gap insulator.

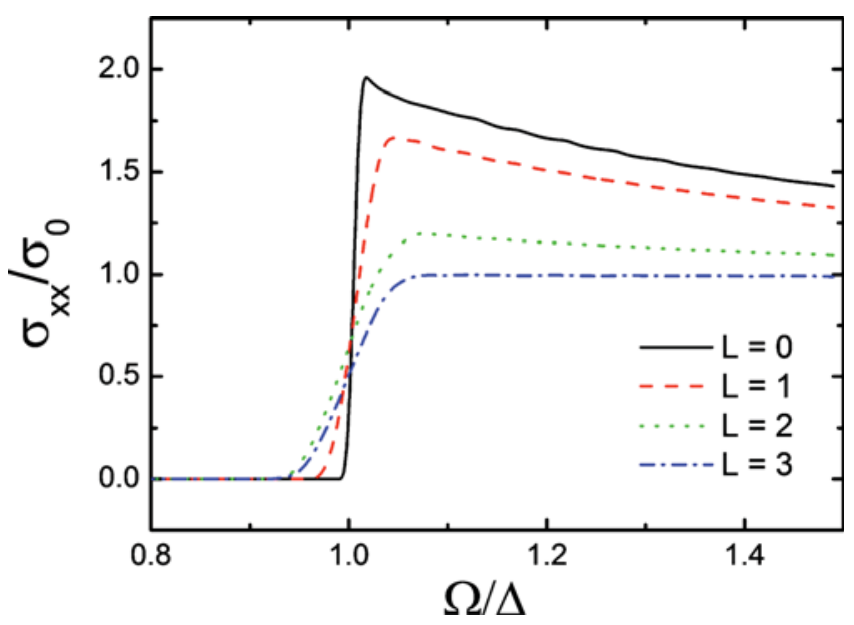

FIG. 4. (Color online) The low energy optical conductivity of all four $S$ $=4$ single layer structures. As the band gap decreases, the onset magnitude decreases, approaching $\sigma_{0}$ for $N_{A}=N_{B}$. However, despite the vanishing band gap, transitions for energies much below $\Delta_{H}$ are still forbidden.
This retained exclusion of interband transitions despite a finite density of states makes the low energy carriers in semihydrogenated graphene particularly robust. This should be an important factor in determining the transport properties of this material.

We have probed the electronic and optical properties of a realistic model of semihydrogenated graphene. We predict that the most likely material to form when a single side of graphene is exposed to hydrogen is an ordered array of Aand B-hydrogenated domains. In this case, for single layers, the band gap predicted for semihydrogenated graphene should disappear, or should be smaller than the actual on-site energy of hydrogenated carbon, $\Delta_{H}$. However, we also predict that the vertical onset energy should be approximately equal to $\Delta_{H}$, but will no longer be vertical and of magnitude $2 \sigma_{0}$, but may be as low as $\sigma_{0}$ and quite shallow. In this way, semihydrogenated single layer graphene behaves as a zerogap insulator. The robust low energy Dirac states for $N_{A}$ $\approx N_{B}$ open an interesting question of the transport properties of carriers in semihydrogenated single layer graphene.

This work is supported by the Australian Research Council (DP0879151).

${ }^{1}$ A. H. Castro Neto, F. Guinea, N. M. R. Peres, K. S. Novoselov, and A. K. Geim, Rev. Mod. Phys. 81, 109 (2009).

${ }^{2}$ K. S. Novoselov, A. K. Geim, S. V. Morozov, D. Jiang, M. I. Katsnelson, I. V. Grigorieva, S. V. Dubonos, and A. A. Firsov, Nature (London) 438, 197 (2005).

${ }^{3}$ C. Berger, Z. Song, X. Li, X. Wu, N. Brown, C. Naud, D. Mayou, T. Li, J. Hass, A. N. Marchenkov, E. H. Conrad, P. N. First, and W. A. de Heer, Science 312, 1191 (2006).

${ }^{4}$ D. C. Elias, R. R. Nair, T. M. G. Mohiuddin, S. V. Morozov, P. Blake, M. P. Halsall, A. C. Ferrari, D. W. Boukhvalov, A. K. Geim, and K. S. Novoselov, Science 323, 610 (2009).

${ }^{5}$ S. Lebègue, M. Klintenberg, O. Eriksson, and M. I. Katsnelson, Phys. Rev. B 79, 245117 (2009).

${ }^{6}$ J. Zhou, M. M. Wu, X. Zhou, and Q. Sun, Appl. Phys. Lett. 95, 103108 (2009).

${ }^{7}$ J. Zhou, Q. Wang, Q. Sun, X. S. Chen, Y. Kawazoe, and P. Jena, Nano Lett. 9, 3867 (2009).

${ }^{8}$ D. Xiao, W. Yao, and Q. Niu, Phys. Rev. Lett. 99, 236809 (2007).

${ }^{9}$ L. Chen, Z. Ma, and C. Zhang, Appl. Phys. Lett. 96, 023107 (2010).

${ }^{10}$ R. R. Nair, P. Blake, A. N. Grigorenko, K. S. Novoselov, T. J. Booth, T. Stauber, N. M. R. Peres, and A. K. Geim, Science 320, 1308 (2008).

${ }^{11}$ C. Zhang, L. Chen, and Z. Ma, Phys. Rev. B 77, 241402 (2008).

${ }^{12}$ T. Stauber, N. M. R. Peres, and A. K. Geim, Phys. Rev. B 78, 085432 (2008).

${ }^{13}$ A. A. Avetisyan, B. Partoens, and F. M. Peeters, Phys. Rev. B 79, 035421 (2009).

${ }^{14}$ J. Cserti, A. Csordas, and G. David, Phys. Rev. Lett. 99, 066802 (2007).

${ }^{15}$ T. G. Pedersen, C. Flindt, J. Pedersen, A.-P. Jauho, N. A. Mortensen, and K. Pedersen, Phys. Rev. B 77, 245431 (2008).

${ }^{16}$ A. R. Wright and C. Zhang, Appl. Phys. Lett. 95, 163104 (2009).

${ }^{17}$ F. M. D. Pellegrino, G. G. N. Angilelle, and R. Pucci, Phys. Rev. B 81, 035411 (2010).

${ }^{18}$ A. R. Wright, X. G. Xu, J. C. Cao, and C. Zhang, Appl. Phys. Lett. 95, 072101 (2009).

${ }^{19}$ J. Liu, A. R. Wright, C. Zhang, and Z. Ma, Appl. Phys. Lett. 93, 041106 (2008).

${ }^{20}$ A. R. Wright, J. C. Cao, and C. Zhang, Phys. Rev. Lett. 103, 207401 (2009).

${ }^{21}$ G. D. Mahan, Many-Particle Physics (Kluwer Academic, New York, 2000). 